\title{
Synthesis of Novel Fluorine-Containing Poly(aryl ether nitrile)s Derived from 2,3,4,5,6-Pentafluorobenzonitrile
}

\author{
Kunio KimURA, Yumi TABUCHI, Ai NishichI, Yuhiko YamASHITA, \\ Yasunori OKUMURA, ${ }^{*}$ and Yoshimitu SAKAGUCHI** \\ Faculty of Environmental Science and Technology, Okayama University, \\ 3-1-1 Tsushima-naka, Okayama 700-8530, Japan \\ *Tsukuba Research Laboratory, Nippon Shokubai Co., Ltd., 1-25-12 \\ Kannondai, Tsukuba 305-0856, Japan \\ ${ }^{* *}$ TOYOBO Research Center Co. Ltd., 1-1 Katata 2-Chome, \\ Ohtsu 520-0292, Japan
}

(Received November 13, 2000; Accepted December 26, 2000)

\begin{abstract}
Pentafluorobenzonitrile (PFBN) is a valuable intermediate and it is available as a commercial product. In this paper, synthesis and characterization of novel fluorinated poly(ether nitrile)s (PEN) derived from PFBN is reported. Novel fluorinated PENs containing fluorine atoms in the main chain and pendant phenoxy group (2F-PEN) are synthesized by aromatic nucleophilic substitution reaction, of which $M_{\mathrm{n}}$ ranges from $1.77 \times 10^{4}$ to $5.16 \times 10^{4}$. The obtained PENs show excellent solubilities in common solvents, and 2F-PEN(6FBA) and 2F-PEN(DPE) can be cast as tough transparent films. These polymers have high thermal stabilities with the $5 \%$ weight loss temperature in the range 509$562^{\circ} \mathrm{C}$ and the $T_{\text {g }}$ of $142-235^{\circ} \mathrm{C}$, which is highly dependent on the structures.

KEY WORDS Poly(ether nitrile) / Fluorine-Containing Polymer / 2,3,4,5,6-Pentafluorobenzonitrile / Nucleophilic Aromatic Substitution / Thermal Stability / High $T_{\mathrm{g}} /$ Solubility /
\end{abstract}

The high-performance fluorinated polymers have been receiving considerable attention as interesting advanced materials for applications as films, coatings for optical and microelectronics devices, gas separation membranes and so on. ${ }^{1-3}$ The incorporation of fluorine atoms into polymer chains leads to polymers with increased solubility, flame resistance, thermal stability and glass transition temperature, while also leading to decreased color, crystallinity, dielectric constant and moisture absorption. Owing these advantages, poly(aryl ether ketone)s (PEK) containing hexafluoroisopropylidene units had been prepared and studied for use in aerospace and electronic applications. ${ }^{4}$ Recently, PEKs containing 2,3,5,6tetrafluoro-1,4-phenylene moieties were synthesized from perfluorobenzophenone. ${ }^{5}$

2,3,4,5,6-Pentafluorobenzoic acid (PFBA) is a valuable intermediate for pharmaceuticals, pesticides, perfumes, cosmetics and so on, and it is available as a commercial product. The authors had reported the synthesis and characterization of novel fluorinated PEKs containing 2,3,5,6-tetrafluoro-1,4-phenylene moieties derived from PFBA.,7 These fluorinated PEKs showed excellent thermal stability and solubility into common organic solvents. Moreover, the films of these PEKs possess outstanding transparency and low dielectric constants. Therefore they are expected to use a optical and electronic materials and the application study is currently underway.

2,3,4,5,6-Pentafluorobenzonitrile (PFBN) is a raw material of PFBA and its commercial availability is much higher than PFBA. 2,6-Difluorobenzonitrile is commonly used as a monomer for poly(ether nitrile)s $(\mathrm{PEN})^{8}$ and other types of fluorinated PENs had been previously synthesized from 2,4,5,6-tetrafluoroisophthalonitrile and bisphenols by interfacial polycondensation. ${ }^{9}$ However, polymers derived from PFBN have not be reported to our knowledge. In this paper, synthesis and characterization of novel fluorinated PEN derived from PFBN is reported.

\section{EXPERIMENTAL}

\section{Materials}

PFBN was a gift from Nippon Shokubai Co. Ltd. Phenol, 2,2-Bis ( 4-hydroxyphenyl ) -1,1,1,3,3,3-hexafluoropropane (6FBA), 2,2-bis (4-hydroxyphenyl)propane (BisA), hydroquinone (HQ), phenolphthalein (PP), 4,4'dihydroxydiphenyl ether ( DPE ), 1,4-bis ( 4hydroxyphenyl ) cyclohexane ( CHB ) and 4,4'dihydroxybiphenyl (BP) were obtained from Aldrich Chemical Co., Inc. 9,9-bis(4-hydroxyphenyl)fluorene (HF) was obtained from Kennedy and Klim. Co., Inc. Bisphenols were purified by recrystallization. $N, N$ dimethylacetamide (DMAc), and $N$-methyl-2pyrrolidinone (NMP) were purchased from Aldrich Chemical Co. Inc. and used as received.

\section{Synthesis of 4-Phenoxy-2,3,5,6-tetrafluorobenzonitrile (PTFB)}

Into a $100 \mathrm{~mL}$ of three-neck flask equipped with a Dean-Stark trap, a condenser and a nitrogen inlet tube were placed $5.0 \mathrm{~g}$ of phenol $(0.053 \mathrm{~mol}), 3.7 \mathrm{~g}$ of potassium carbonate $(0.027 \mathrm{~mol}), 60 \mathrm{~mL}$ of $\mathrm{NMP}$ and $10 \mathrm{~mL}$ of toluene. The mixture was refluxed for $2 \mathrm{~h}$ with eliminating by-produced water by azeotrop distillation and then toluene was distilled over. The mixture was allowed to cool at $80^{\circ} \mathrm{C}$ and then $10.3 \mathrm{~g}$ of PFBN $(0.053 \mathrm{~mol})$ was added into the mixture. The reaction was allowed to keep at $80^{\circ} \mathrm{C}$ for $12 \mathrm{~h}$. The reaction mixture was allowed to cool to room temperature and then poured into 200 $\mathrm{mL}$ of water, which was extracted with dichloromethane. The organic layer was collected, washed with water, 
dried over sodium sulfate, filtered and evaporated. The unreacted PFBN was stripped off by distillation under reduced pressure and then the residual solids were recrystallized from ethanol. White crystals of 4-phenoxy2,3,5,6-tetrafluorobenzonitrile (PTFB) were obtained at $5.4 \mathrm{~g}(38 \%)$. The product characteristics were as follows; $T_{\mathrm{m}}: 67-68^{\circ} \mathrm{C}$, FT-IR $(\mathrm{KBr})\left(\mathrm{cm}^{-1}\right): 3047,2932,2243$, $1651,1593,1492,1437,1319,1203,1169,1119,1074$, $995,957,920,781,752,735,687$.

\section{Polymerization}

Polymerization of PTFB with 6FBA is given as a typical example as follows. $1.26 \mathrm{~g}$ of $6 \mathrm{FBA}$ ( $3.74 \mathrm{mmol}), 0.57$ $\mathrm{g}$ of potassium carbonate $(4.12 \mathrm{mmol}), 8 \mathrm{~mL}$ of NMP and $10 \mathrm{~mL}$ of toluene were placed into a $50 \mathrm{~mL}$ of three-neck flask equipped with Dean-Stark trap, condenser, magnet stirrer and nitrogen inlet tube. The mixture was refluxed for $2 \mathrm{~h}$ with eliminating by-produced water by azeotrop distillation and then toluene was distilled over. The mixture was allowed to cool at $80^{\circ} \mathrm{C}$ and then $1.0 \mathrm{~g}$ of PTFB (3.74 mmol) was added into the mixture. The polymerization was allowed to keep at $80^{\circ} \mathrm{C}$ for $20 \mathrm{~h}$. The reaction mixture was allowed to cool to room temperature and poured into rapidly stirred deionized water containing $1 \mathrm{wt} \%$ acetic acid to precipitate the polymer. The polymer was isolated by filtration, washed twice with deionized water and dried. The polymer $\{2 \mathrm{~F}-\mathrm{PEN}(6$ FBA)\} was purified by the reprecipitation of DMAc solution to methanol. The product characteristics were as follows. $T_{\mathrm{g}}: 163^{\circ} \mathrm{C}$, FT-IR $(\mathrm{KBr})\left(\mathrm{cm}^{-1}\right): 3059,2239,1593$, 1508, 1475, 1230, 1180, 1136, 1001, 962, 933, 831, 752, 696.

\section{Characterization}

NMR spectra were recorded on an Varian Unity-500 operating at $500 \mathrm{MHz}\left({ }^{1} \mathrm{H}\right), 125 \mathrm{MHz}\left({ }^{13} \mathrm{C}\right)$ and $470 \mathrm{MHz}$ $\left({ }^{19} \mathrm{~F}\right) . \mathrm{CDCl}_{3}$ and DMSO- $d_{6}$ were used as the measuring solvents for monomer and polymers. In some cases, hexafluoroisopropanol was added into the solution to dissolve the samples completely. 4,4'-Difluorobenzophenone was used as an internal standard for ${ }^{19} \mathrm{~F}$ NMR measurements. FT-IR measurements were performed on JASCO FT-IR 350. Glass transition temperature $\left(T_{\mathrm{g}}\right)$ was measured on a Perkin-Elmer DSC 7 at a scanning rate of $20^{\circ} \mathrm{C} \mathrm{min}{ }^{-1}$ in nitrogen atmosphere. Temperature and heat flow were calibrated using indium and zinc as the standards. Thermal stability was measured on a Perkin-Elmer TGA 7 at a heating rate of $20^{\circ} \mathrm{C} \min ^{-1}$ in nitrogen atmosphere. Reduced viscosity measurements were determined by using an OstwaldFenske viscometer in DMAc at a concentration of $0.5 \mathrm{~g}$ $\mathrm{dL}^{-1}$ and $25^{\circ} \mathrm{C}$. Molecular weights were measured by TOSOH 8020 GPC. NMP was used as an eluent and column temperature was $40^{\circ} \mathrm{C}$. Molecular weights were calculated with polystyrene standard. Dynamic mechanical measurement was performed on Orientech Rheovibron DDV-II-EP at $110 \mathrm{~Hz}$ with a scanning rate of $2^{\circ} \mathrm{C} \mathrm{min}-1$.

\section{RESULTS AND DISCUSSION}

\section{Monomer Synthesis}

PFBN has three reactive positions for nucleophilic aromatic substitution reaction, that is, two ortho

Polym. J., Vol. 33, No. 3, 2001 posisions and one para position to cyano group. Multifunctional PFBN leads to crosslinking reaction during synthesis of PEN. Therefore, PTFB is designed in which the para position of PFBN is substituted by phenoxy group, and hence linear PENs can be obtained by the reaction between PTFB and bisphenols at ortho positions to cyano group of PTFB. PTFB was synthesized from PFBN and phenoxide by aromatic nucleophilic substitution as shown in Scheme 1. When the reaction was carried out over $100^{\circ} \mathrm{C}$, phenoxide substituted at not only para position but ortho position. Hereby a small amount of diphenoxybenzonitrile was contaminated. The reaction was performed at $80^{\circ} \mathrm{C}$ to yield the only para substituted benzonitrile even though the yield became lower which was $38 \%$. The chemical structure of PTFB was confirmed by ${ }^{1} \mathrm{H},{ }^{13} \mathrm{C},{ }^{19} \mathrm{~F}$ NMR, and FT-IR spectroscopy. The spectrum of ${ }^{19} \mathrm{~F},{ }^{1} \mathrm{H}$ NMR, and FT-IR are illustrated in Figures $1-3$. Two peaks are observed in ${ }^{19} \mathrm{~F}$ NMR spectrum at -140.7 and $-122.2 \mathrm{ppm}$ corresponding to two meta fluorines and two ortho fluorines to cyano group, respectively. In ${ }^{1} \mathrm{H}$ NMR spectrum, there exists three peaks at 7.36, 7.19, and $6.99 \mathrm{ppm}$ corresponding to two meta protons, one para proton and two ortho protons to ether linkage, respectively. The peak of cyano group is clearly observed at $2243 \mathrm{~cm}^{-1}$ in FT-IR spectrum.

\section{Polymer Synthesis}

Novel eight PENs are synthesized from PTFB (2FPEN) as shown in Scheme 2. Nucleophilic aromatic substitution polymerizations are typically performed in a high-boiling aprotic polar solvent in the presence of a base at high temperature. Polymerizations are carried out in NMP. The solids compositions are maintained at $25 \%$. The potassium carbonate is used to convert the bisphenol into the more reactive anion. Irrespective of

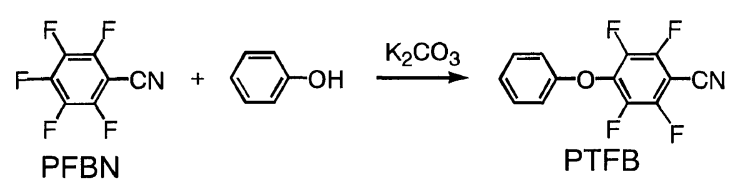

Scheme 1. Monomer synthesis.
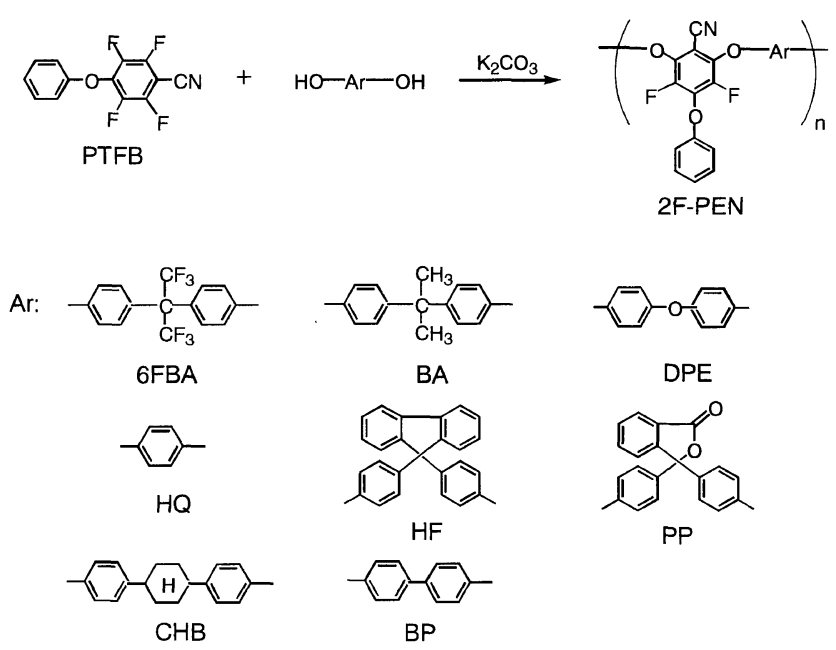

PP

Scheme 2. Synthesis of fluorine-containing poly(ether nitrile)s. 


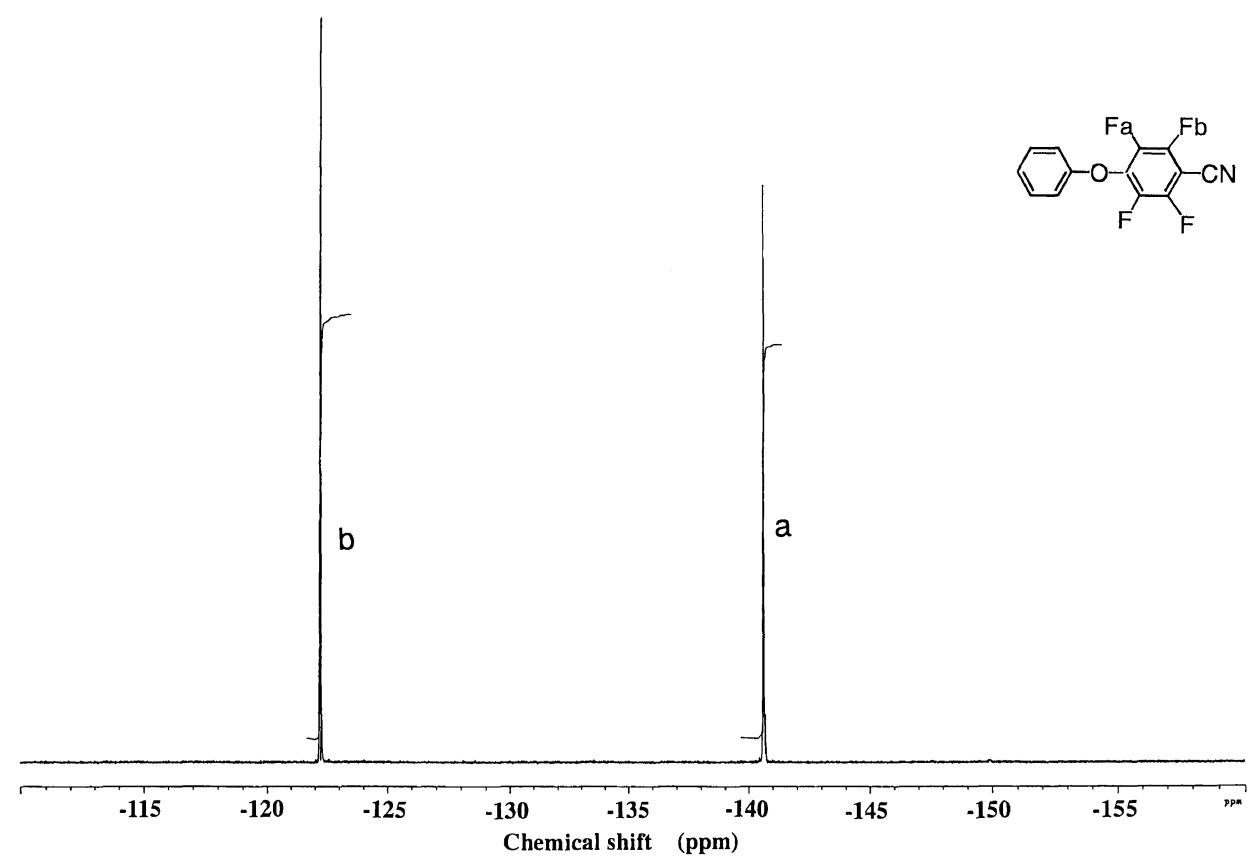

Figure 1. ${ }^{19} \mathrm{~F}$ NMR spectrum of PTFB.

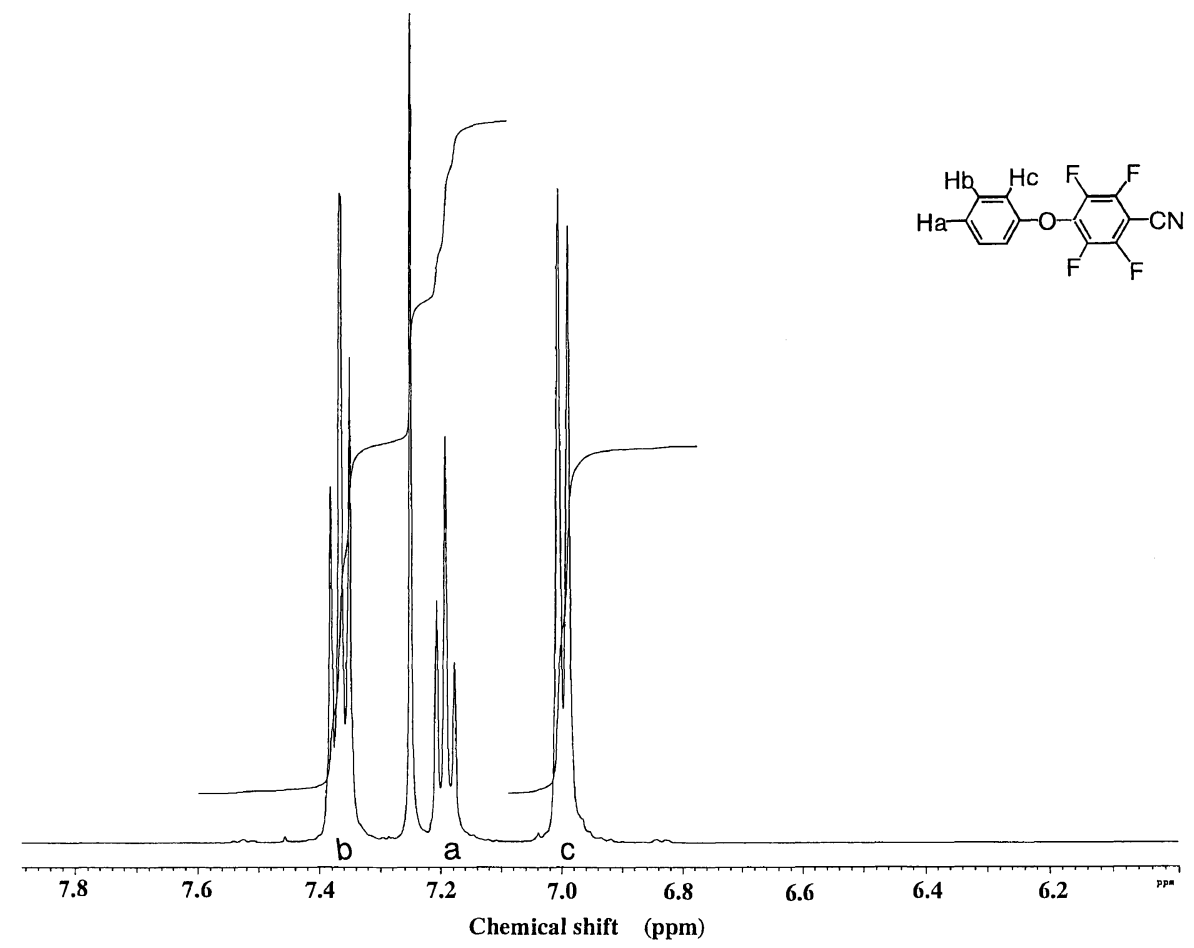

Figure 2. ${ }^{1} \mathrm{H}$ NMR spectrum of PTFB.

the solvents, toluene is used during the initial stages of the polymerizations to remove water generated by phenoxide formation as a toluene azeotrope.

Table I summarizes the results of polymerization. The polymerization of PTFB with $6 \mathrm{FBA}$ over $100^{\circ} \mathrm{C}$ yielded gels due to the crosslinking reaction, in which bisphenoxide substitutes at not only ortho position but metha position to cyano group in PTFB. In order to avoid the gelation, the polymerization was carried out at $80^{\circ} \mathrm{C}$. The soluble 2F-PEN (6FBA) are obtained, of which the viscosity is $0.66 \mathrm{dL} \mathrm{g}^{-1}$. The polymerization of PTFB with other bisphenols was also carried out at $80^{\circ} \mathrm{C}$. The soluble 2F-PENs were obtained, of which the viscosities range from 0.22 to $1.27 \mathrm{dL} \mathrm{g}^{-1}$. The $\mathrm{M}_{\mathrm{n}}$ s of these $2 \mathrm{~F}$ PENs range from $1.77 \times 10^{4}$ to $5.16 \times 10^{4}$ and the molecular weight distribution $M_{\mathrm{w}} / M_{\mathrm{n}}$ are 2.02-6.81. Among them, 2F-PEN (6FBA) and 2F-PEN (DPE) can be cast from toluene solutions into tough transparent films. The polymer structures are analyzed by ${ }^{19} \mathrm{~F},{ }^{1} \mathrm{H}$ NMR, and FT-IR. ${ }^{19} \mathrm{~F}$ and ${ }^{1} \mathrm{H}$ NMR spectra of $2 \mathrm{~F}$-PEN (6FBA) are 


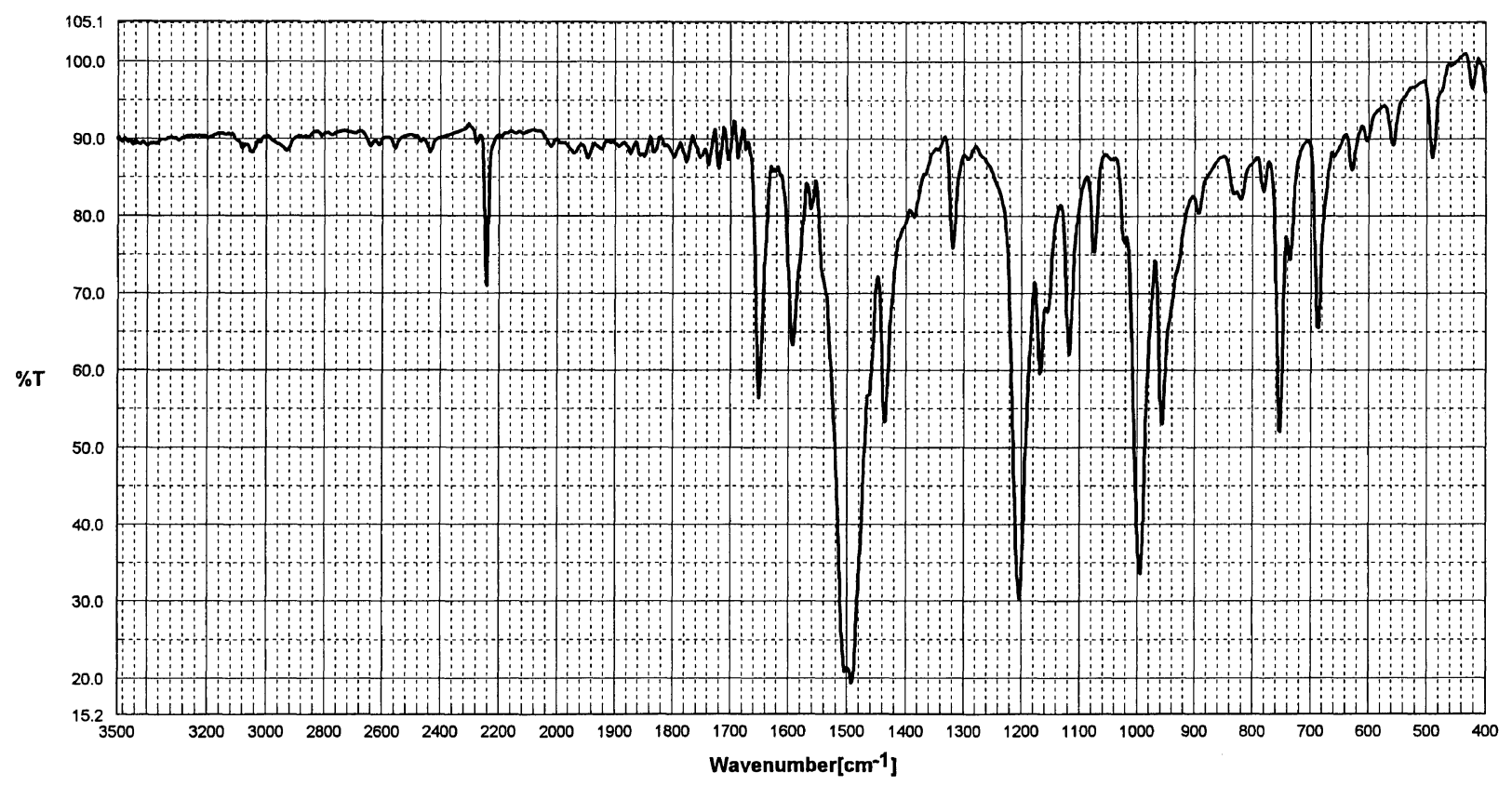

Figure 3. FT-IR spectrum of PTFB.

Table I. Polymerization of 4-phenoxy-2,3,5,6-tetrafluorobenzonitrile with various bisphenols ${ }^{\mathrm{a}}$

\begin{tabular}{|c|c|c|c|c|c|c|c|}
\hline Polymer Code ${ }^{b}$ & $\frac{\text { Temp. }}{{ }^{\circ} \mathrm{C}}$ & $\frac{\text { Con. }}{\text { wt/vol\% }}$ & $\frac{\text { Yield }^{\mathrm{c}}}{\%}$ & $\frac{\eta \mathrm{sp} / \mathrm{c}^{\mathrm{d}}}{\mathrm{dL} \mathrm{g}^{-1}}$ & $\frac{M_{\mathrm{n}}^{\mathrm{e}}}{\left(\times 10^{4}\right)}$ & $\frac{M_{\mathrm{w}}{ }^{\mathrm{e}}}{\left(\times 10^{4}\right)}$ & $M_{\mathrm{w}} / M_{\mathrm{n}}^{\mathrm{e}}$ \\
\hline 2F-PEN-6FBA & 80 & 25 & 86.0 & 0.66 & 5.16 & 29.07 & 5.63 \\
\hline 2F-PEN-PP & 80 & 25 & 94.9 & 0.44 & 2.82 & 11.22 & 3.98 \\
\hline 2F-PEN-BisA & 80 & 25 & 86.8 & 0.35 & 2.19 & 6.34 & 2.90 \\
\hline 2F-PEN-HF & 80 & 25 & 93.5 & 0.32 & 1.92 & 7.96 & 4.13 \\
\hline 2F-PEN-HQ & 80 & 25 & 85.5 & 0.34 & 2.00 & 4.03 & 2.02 \\
\hline 2F-PEN-CHB & 80 & 25 & 87.4 & 0.22 & 1.77 & 3.86 & 2.17 \\
\hline 2F-PEN-DPE & 80 & 25 & 87.1 & 1.27 & 2.98 & 20.33 & 6.81 \\
\hline 2F-PEN-BP & 80 & 25 & 90.7 & 0.57 & 3.17 & 7.57 & 2.39 \\
\hline
\end{tabular}

${ }^{\mathrm{a}}$ Polymerizations were carried out in NMP for $20 \mathrm{~h} .{ }^{\mathrm{b}}$ Polymer codes are shown in Scheme $2 .{ }^{\mathrm{c}}$ After purification by reprecipitation. ${ }^{\mathrm{d}}$ Viscosities were measured in DMAc at a concentration of $0.5 \mathrm{~g} \mathrm{dL}^{-1}$ and $25^{\circ} \mathrm{C}$. ${ }^{e}$ Measured by GPC. Molecular weights are relative to polystyrene standards.

Table II. Solubility of 2F-PENs

\begin{tabular}{|c|c|c|c|c|c|c|c|c|}
\hline \multirow{2}{*}{ Polymer code ${ }^{a}$} & \multicolumn{8}{|c|}{ 2F-PEN } \\
\hline & $6 \mathrm{FBA}$ & $\mathrm{PP}$ & BisA & $\mathrm{HF}$ & $\mathrm{HQ}$ & $\mathrm{CHB}$ & DPE & $\mathrm{BP}$ \\
\hline \multicolumn{9}{|l|}{ Solubility $^{\mathrm{b}}$} \\
\hline NMP & ++ & ++ & ++ & ++ & ++ & ++ & ++ & ++ \\
\hline DMAc & ++ & ++ & ++ & ++ & ++ & ++ & ++ & ++ \\
\hline Chloroform & ++ & ++ & ++ & ++ & + & ++ & ++ & ++ \\
\hline THF & ++ & ++ & ++ & ++ & ++ & ++ & ++ & ++ \\
\hline Toluene & ++ & ++ & ++ & ++ & +- & ++ & ++ & - \\
\hline
\end{tabular}

${ }^{a}$ Polymer codes are shown in Scheme $2 .{ }^{\mathrm{b}}++$ Soluble at $25^{\circ} \mathrm{C},+$ soluble at $100^{\circ} \mathrm{C},+-$ partially soluble or swelling, - insoluble.

illustrated in Figures 4 and 5 as examples. Three peaks are observed in ${ }^{19} \mathrm{~F}$ NMR spectrum at -54.3 and -128.5 ppm corresponding to six aliphatic fluorines and two aromatic fluorines at meta position to cyano group, respectively. In ${ }^{1} \mathrm{H}$ NMR spectrum, there exists two peaks at $6.9-7.4 \mathrm{ppm}$ corresponding to five different aromatic proton. The peak of cyano group is clearly observed at $2239 \mathrm{~cm}^{-1}$ and the peak of ether linkage appear at 1230 $\mathrm{cm}^{-1}$ in FT-IR spectrum of $2 \mathrm{~F}$-PEN (6FBA) as shown in Figure 6. It becomes clear from these results that nucleophilic aromatic substitution takes place at both ortho positions to cyano group of PTFB and 2F-PEN (6FBA) has a linear structure without crosslinking.

\section{Characteristics}

Qualitative solubilities of the new 2F-PENs were determined in excess of solvents. Table II summarizes the results of solubilities. The 2 F-PENs show good solubilities in aprotic polar solvents. Especially, 2F-PEN (6 FBA), 2F-PEN (PP), 2F-PEN (BisA), 2F-PEN (HF), 2FPEN (CHB), and 2F-PEN (DPE) exhibit wider solubilities in chloroform and toluene. PEN containing no fluo- 


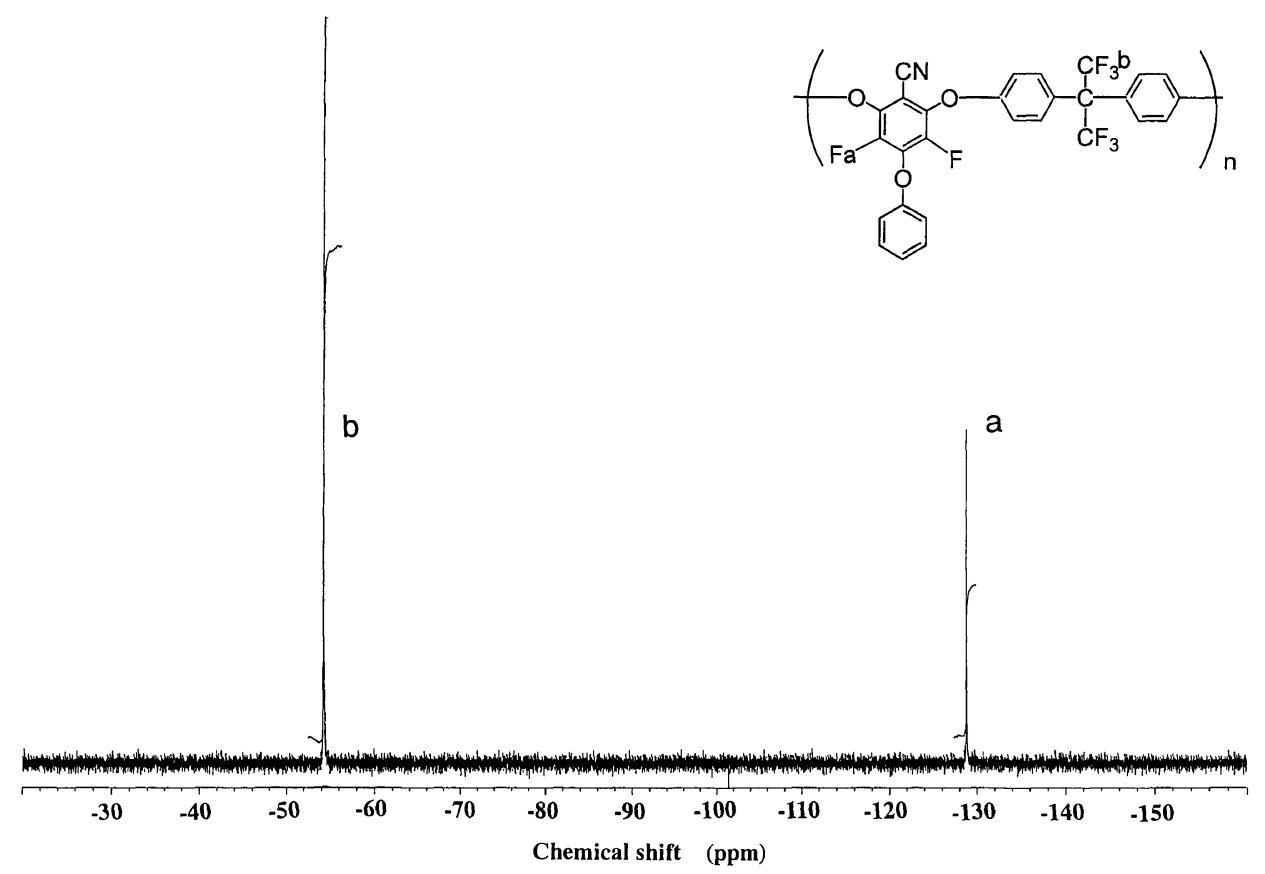

Figure 4. ${ }^{19} \mathrm{~F}$ NMR spectrum of $2 \mathrm{~F}-\mathrm{PEN}$ (6FBA).

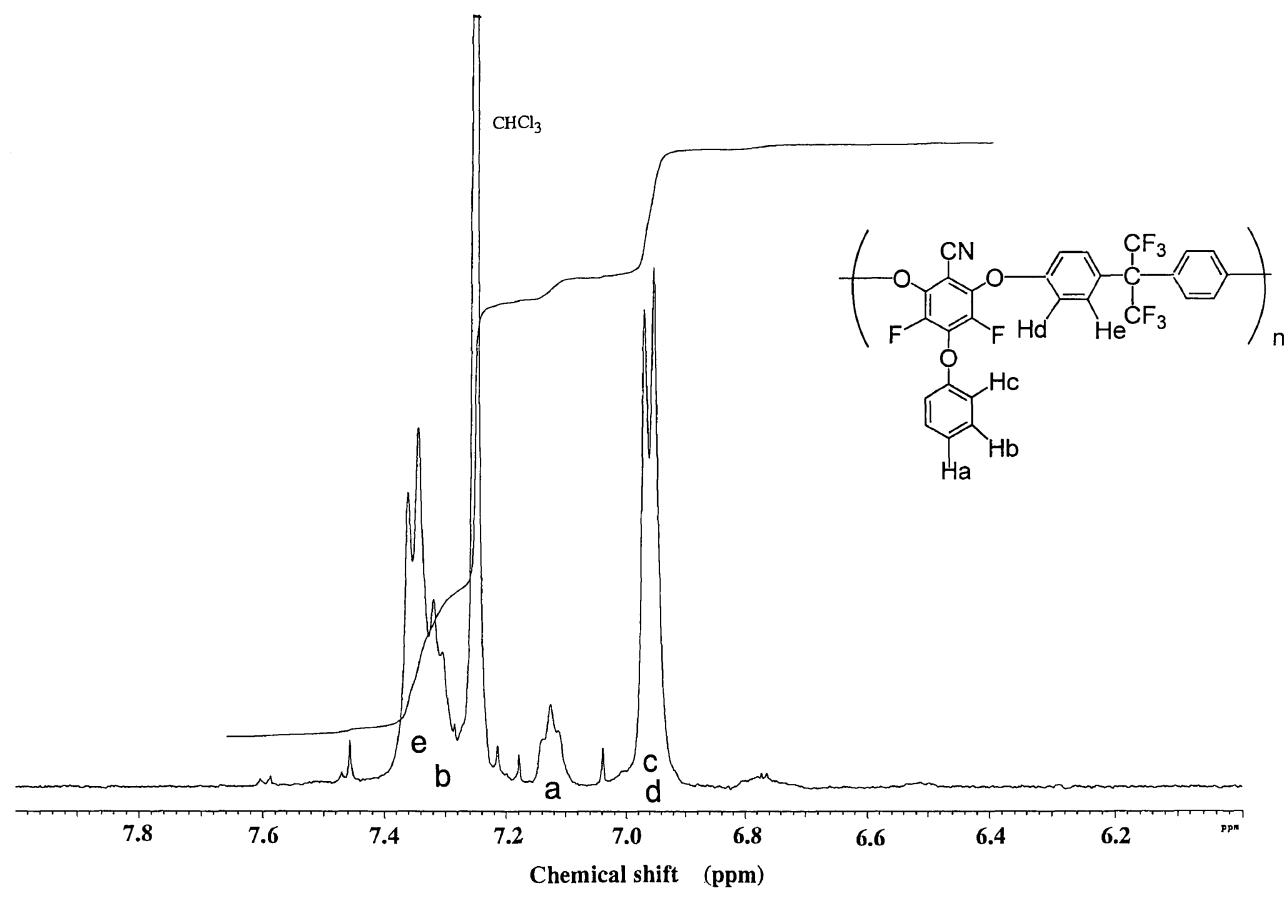

Figure 5. $\quad{ }^{1} \mathrm{H}$ NMR spectrum of $2 \mathrm{~F}-\mathrm{PEN}$ (6FBA).

rine atoms is soluble only in polar solvents such as NMP and DMAc. ${ }^{8}$ The fluorine atoms, 1,3-connection of the back bone and furthermore, bulky phenoxy groups are the reasons to enhance the solubility very much.

Thermal properties are summarized in Table III. The $T_{\mathrm{g}}$ of the obtained fluorine-containing PENs is in the range $142-235^{\circ} \mathrm{C}$ depending on the chemical structures. Among them, 2F-PEN (HF) and 2F-PEN (PP) possess significantly high $T_{\mathrm{g}}$ and it is attributed to the presence of the large cyclic side groups in the polymer chain which is consistent with the previous work. ${ }^{5}$ 2F-PENs obtained in this study do not show the endothermic peak on DSC profiles corresponded to melting and they are amorphous polymers.

Thermal stability was measured by TGA. The 5\% weight loss in a nitrogen atmosphere is quite high, which is in the range $509-562^{\circ} \mathrm{C}$. The char yields at $600^{\circ} \mathrm{C}$ in a nitrogen atmosphere are over $60 \%$ and those of 2F-PEN (HF), 2F-PEN (DPE), and 2F-PEN (BP) are over $80 \%$. Among them, 2F-PEN (HF) exhibits outstanding thermal stability with the $5 \%$ weight loss temperature of $562^{\circ} \mathrm{C}$ and the char yield at $800^{\circ} \mathrm{C}$ of $70 \%$. It is 


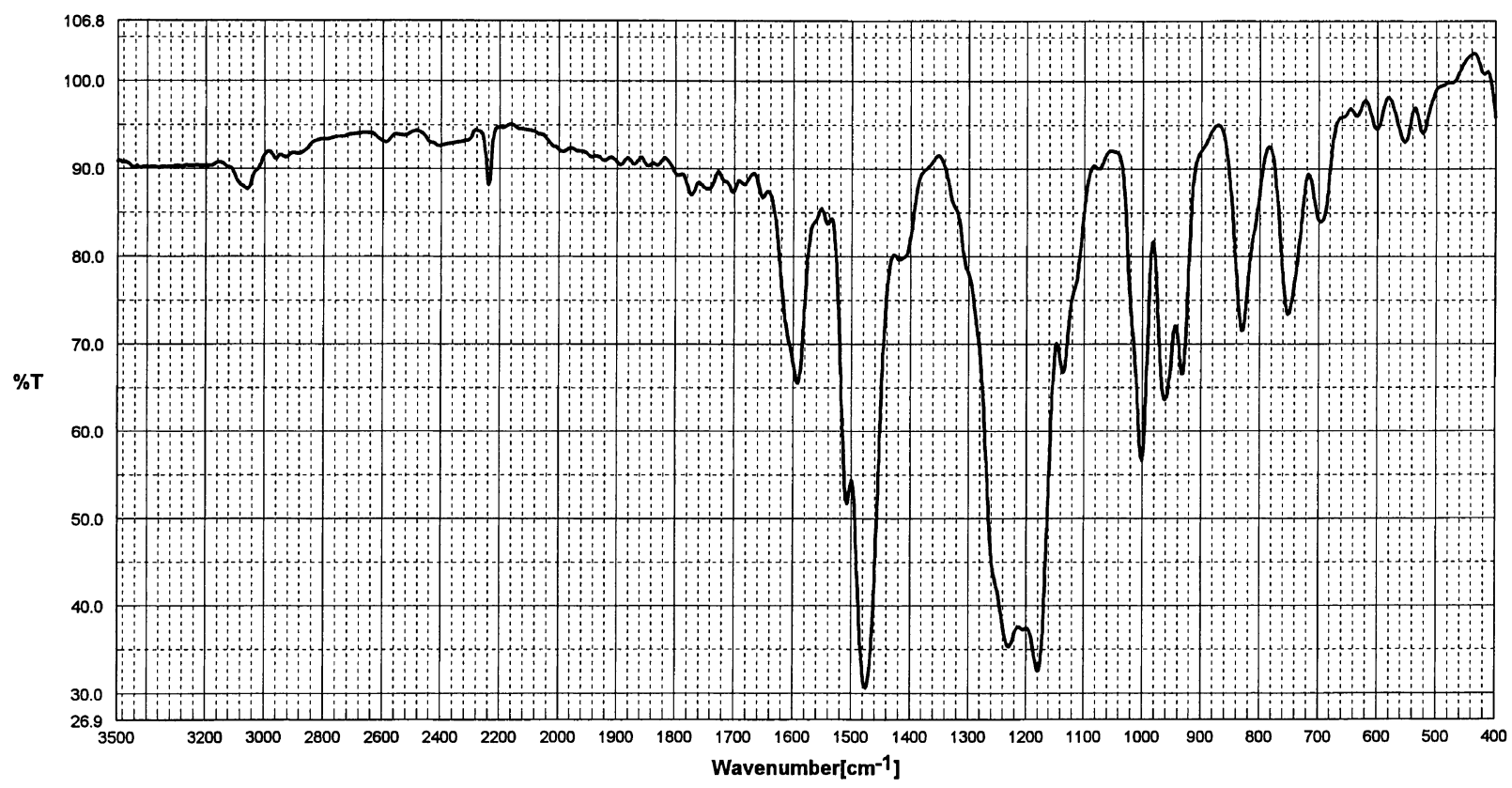

Figure 6. FT-IR spectrum of 2F-PEN (6FBA).

Table III. Thermal properties of 2F-PENs

\begin{tabular}{|c|c|c|c|c|c|c|}
\hline \multirow{2}{*}{ Polymer Code ${ }^{a}$} & \multirow{2}{*}{$\frac{T_{\mathrm{g}}^{\mathrm{b}}}{{ }^{\circ} \mathrm{C}}$} & \multirow{2}{*}{$\frac{5 \mathrm{wt} \% \operatorname{loss}^{\mathrm{c}}}{{ }^{\circ} \mathrm{C}}$} & \multirow{2}{*}{$\frac{10 \mathrm{wt} \% \operatorname{loss}^{\mathrm{c}}}{{ }^{\circ} \mathrm{C}}$} & \multicolumn{3}{|c|}{ Char Yield/wt\% ${ }^{\mathrm{c}}$} \\
\hline & & & & $600^{\circ} \mathrm{C}$ & $700^{\circ} \mathrm{C}$ & $800^{\circ} \mathrm{C}$ \\
\hline 2F-PEN-6FBA & 163.0 & 535 & 561 & 75 & 49 & 29 \\
\hline 2F-PEN-PP & 235.0 & 530 & 554 & 76 & 67 & 62 \\
\hline 2F-PEN-BisA & 143.6 & 531 & 546 & 68 & 60 & 56 \\
\hline 2F-PEN-HF & 232.2 & 562 & 593 & 89 & 75 & 70 \\
\hline 2F-PEN-HQ & 145.9 & 528 & 550 & 73 & 62 & 55 \\
\hline 2F-PEN-CHB & 151.8 & 509 & 522 & 60 & 54 & 51 \\
\hline 2F-PEN-DPE & 142.0 & 533 & 567 & 80 & 60 & 45 \\
\hline 2F-PEN-BP & 179.4 & 558 & 582 & 86 & 73 & 65 \\
\hline
\end{tabular}

${ }^{\mathrm{a}}$ Polymer codes are shown in Scheme $2 .{ }^{\mathrm{b}} \mathrm{DSC}$ was performed with $20^{\circ} \mathrm{C} \mathrm{min}{ }^{-1}$ in nitrogen atmosphere. ${ }^{\mathrm{c}}$ TGA was performed with $20^{\circ} \mathrm{C}$ min $^{-1}$ in nitrogen atmosphere.

well known that the cyano groups afford triazine ring by an addition mechanism ${ }^{10,11}$ and previous study reported that poly(imide-amide)s containing pendant cyano group treated at high temperature lost a good solubility and this insolubility might be due to the formation of triazine rings from cyano groups at high temperature which led to the crosslinked structure. ${ }^{12}$ In the case of 2 F-PENs, char remained at $600^{\circ} \mathrm{C}$ is not completely soluble in polar aprotic solvents. FT-IR measurement of the char shows very slight decrease in the relative intensity of the cyano absorption at $2239 \mathrm{~cm}^{-1}$. But no characteristic absorption for the triazine ring formation could be unambiguously assigned because phenyl and triazine rings have very similar infrared absorption. Very high char yields of 2 F-PENs may attributed to fluorine atoms as well as the formation of the triazine rings. From these results, they possess outstanding thermal stabilities.

Dynamic mechanical property of $2 \mathrm{~F}-\mathrm{PEN}$ (6FBA) is shown in Figure 7. Dynamic modulus at $25^{\circ} \mathrm{C}$ is $1.73 \times$ $10^{10}$ dyne $\mathrm{cm}^{-2}$. It is almost constant up to $130^{\circ} \mathrm{C}$ and then decreased gradually due to $\alpha$ relaxation corresponding to glass transition. $\beta$ relaxation is observed around $80^{\circ} \mathrm{C}$. In poly(ether ether ketone) (PEEK) dynamic mechanical behavior, $\beta$ relaxation was observed

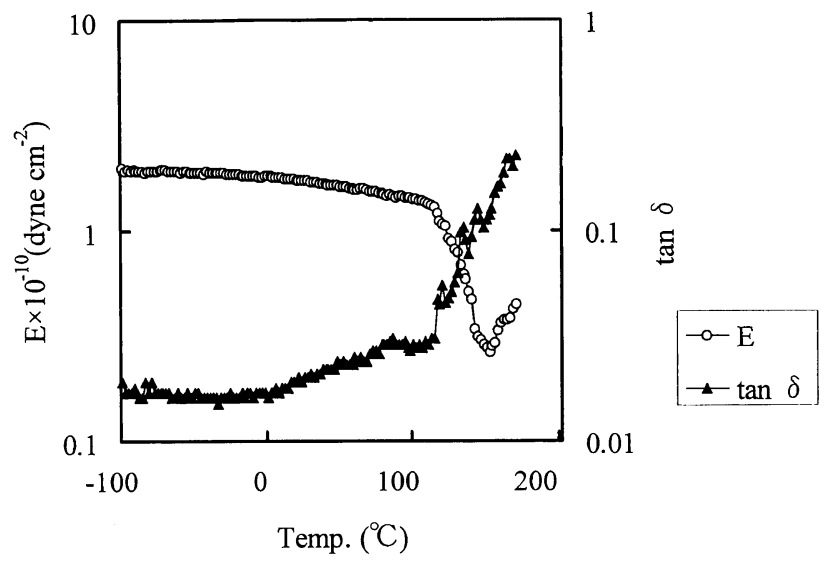

Figure 7. Dynamic mechanical scan of $2 \mathrm{~F}-\mathrm{PEN}(6 \mathrm{FBA})$ at 110 $\mathrm{Hz}$. Scanning rate was $2^{\circ} \mathrm{C} \mathrm{min}^{-1}$.

around $130^{\circ} \mathrm{C}$ and this behavior was interpreted as an effect of water ${ }^{13,14}$ or a counterrotational motion in diphenyl ether moiety. ${ }^{15}$ According to these previous discussions, $\beta$ relaxation of $2 \mathrm{~F}-\mathrm{PEN}$ (6FBA) occurs at lower temperature than that of PEEK and its behavior seems to be attributable to rotational motion of pendant phe- 
noxy group or cyano and fluoro substituted diphenyl ether moiety in polymer chain.

\section{CONCLUSION}

Novel fluorinated PENs containing fluorine atoms in the main chain and pendant phenoxy group are synthesized by aromatic nucleophilic substitution reaction, of which $M_{\mathrm{n}}$ ranges from $1.77 \times 10^{4}$ to $5.16 \times 10^{4}$. The obtained PENs show excellent solubilities in common solvents, and 2F-PEN (6FBA) and 2F-PEN (DPE) can be cast as tough transparent films. These polymers have high thermal stabilities with the $5 \%$ weight loss temperature in the range $509-562^{\circ} \mathrm{C}$ and the $T_{\mathrm{g}}$ of $142-$ $235^{\circ} \mathrm{C}$, which is highly dependent on the structures.

\section{REFERENCES}

1. M. Bruma, J. W. Fitch, and P. E. Cassidy, J. Macromol. Sci., Rev. Macromol. Chem. Phys., C36, 119 (1996).

2. P. E. Cassidy, J. Macromol. Sci., Rev. Macromol. Chem.
Phys., C34, 1 (1994).

3. P. E. Cassidy, T. M. Aminabhavi, and J. M. Farley, J. Macromol. Sci., Rev. Macromol. Chem. Phys., C29, 365 (1989).

4. G. L. Tullos, and P. E. Cassidy, Macromolecules, 24, 6059 (1991).

5. F. W. Mercer, M. M. Fone, V. N. Reddy, and A. A. Goodwin, Polymer, 38, 1989 (1997).

6. K. Kimura, Y. Yamashita, P. E. Cassidy, J. W. Fitch III, V. S. Reddy, and Y. Sakaguchi, Polym. Prepr., Am. Chem. Soc., (Div. Polym. Chem.) 39(2), 790 (1998).

7. K. Kimura, Y. Tabuchi, Y. Yamashita, P. E. Cassidy, J. W. Fitch III, and Y. Okumura, Polym. Adv. Tech., 11(8-12), 757 (2000).

8. S. Matsuo, T. Murakami, and R. Takazawa, J. Polym. Sci., Part A: Polym. Chem., 31, 3439 (1993).

9. M. Shimizu, M. Kakimoto, and Y. Imai, J. Polym. Sci., Part A: Polym. Chem., 25, 2385 (1987).

10. T. M. Keller, J. Polym. Sci., Part A: Polym. Chem., 26, 3199 (1988).

11. T. M. Keller, Polymer, 34, 952 (1993).

12. P. E. Cassidy, private communication.

13. T. Sasuga and M. Hasegawa, Polymer, 26, 501 (1985).

14. T. Sasuga and M. Hasegawa, Polymer, 27, 821 (1986).

15. L. David and S. Etienne, Macromolecules, 25, 4302 (1992). 